\title{
Qualitative Analysis of DoorDash
}

\author{
Yiwen Zhang ${ }^{1, *}$
}

${ }^{1}$ School of Social Sciences, University of California, Irvine, California, United States, 92617

*Corresponding author. Email:yiwez@uci.edu

\begin{abstract}
For the reason that phones and internet have become pervasive recently, online ordering and food delivery service have become more popular. In 1994, the online ordering service for pizzas showed up, and in the 2000s, major pizza chains have mature online ordering service.

This study aims to explore the qualitative analysis of DoorDash by using Michael Porter's five-force model. For DoorDash, its rivalry among existing competitors is strong, and the threat of substitute products is strong. Also, the bargaining power of suppliers is becoming stronger. Meanwhile, the threat of new entrants is small, and the bargaining power of buyers is relatively weak in the American suburbia.
\end{abstract}

Keywords: DoorDash, food delivery, online ordering, Michael Porter's five-force model, Grubhub, Uber Eats

\section{INTRODUCTION}

With the development of technologies, more people began to get used to using applications to order food online. From 2015 to 2020 , the number of food delivery application users have been increasing constantly, as is shown in Figure 1. In 2020, because of the Covid 19, users increased much more than other years.

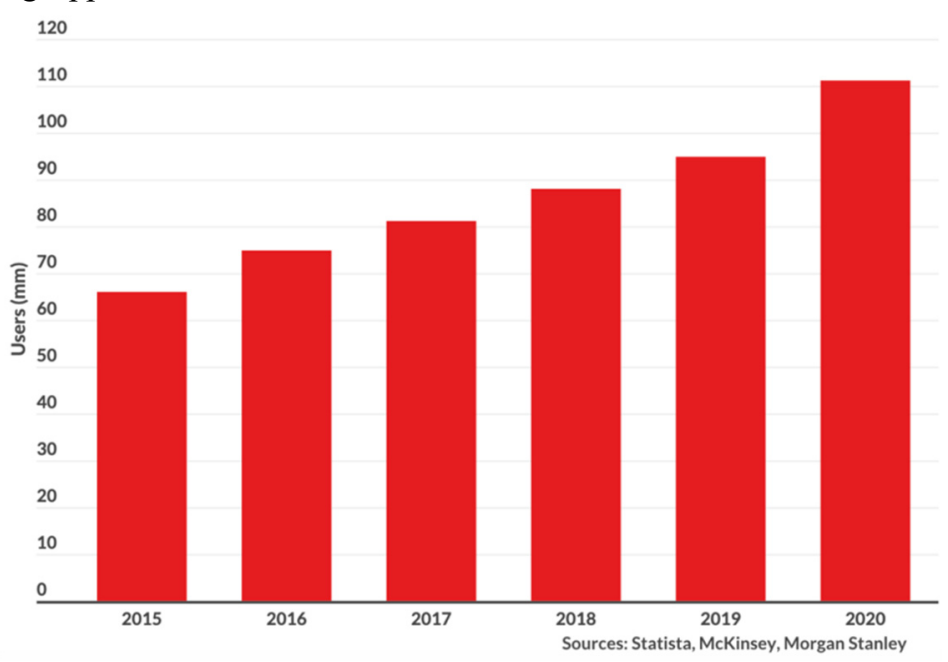

Figure 1: An image of US food delivery App users that are increasing each year from 2015 to 2020.

Also, the food delivery industry became crowded, and many companies are trying to achieve the leading position in this industry. DoorDash is one of the platforms that offer online food ordering and food delivery. It was founded in 2013, and it went public on New York Stock in 2021. However, it is still not earning profits, although in recent years, it had the fastest speed in developing among the delivery companies. Whether DoorDash could begin to earn profits after its rapid development in the quarantine time becomes stimulating and absorbing. 


\section{RIVALRY AMONG EXISTING COMPETITORS}

The rivalry among existing competitors is strong. In the food delivery industry, there are three existing companies that have relatively equal size, which are DoorDash, Grubhub, and Uber Eats. Grubhub was founded with Seamless in the early 2000s, which is the oldest company among the three. For the following ten years, Grubhub and Seamless were dominant in the food delivery industry. However, as the competition in this industry began to become intense in the early 2010s, Grubhub merged with Seamless in 2013, in order to maintain its dominant role in the industry. This decision represented a large and intense rivalry among existing competitors in this industry, and it could also threaten the business of DoorDash. But, Grubhub was acquired by Just Eat Takeaway.com in June 2021.

After they combined, Postmates, DoorDash and Uber Eats sprung up and obtained parts of orders. Uber Eats was competing with DoorDash fiercely. Besides the U.S., Uber Eats expanded its service to many other countries at the beginning few years, but it ended plenty of them in the past few years, including the service in
Korea and India. To retain its position in the industry, Uber Eats made an acquisition of Postmates which is also a food delivery platform. This movement is the same as what Grubhub did, and their purposes were identical. Ultimately, their objectives were using acquisition to consolidate the proportion in the food delivery industry and increase their revenues, and their actions could be threats to DoorDash.

In comparison, DoorDash was the one to be wanted to make an acquisition with. DoorDash was founded in 2013, which is close to the founding year with Uber Eats, but DoorDash developed more rapidly than Uber Eats. It surpasses Grubhub in 2019 because of the pandemic, therefore, Uber Eats tried to merge with DoorDash in order to eliminate this tough competitor. DoorDash didn't compromise and "in 2020, DoorDash was responsible for 45 percent of all food delivery orders" [1]. Figure 2 shows the percentage changes of food delivery market share between Grubhub, DoorDash, Uber Easts, and Postmates from 2016 to 2020 , which shows the fact that the rivalry in food delivery industry began to be intense from 2016 and became more intensely at this present time.

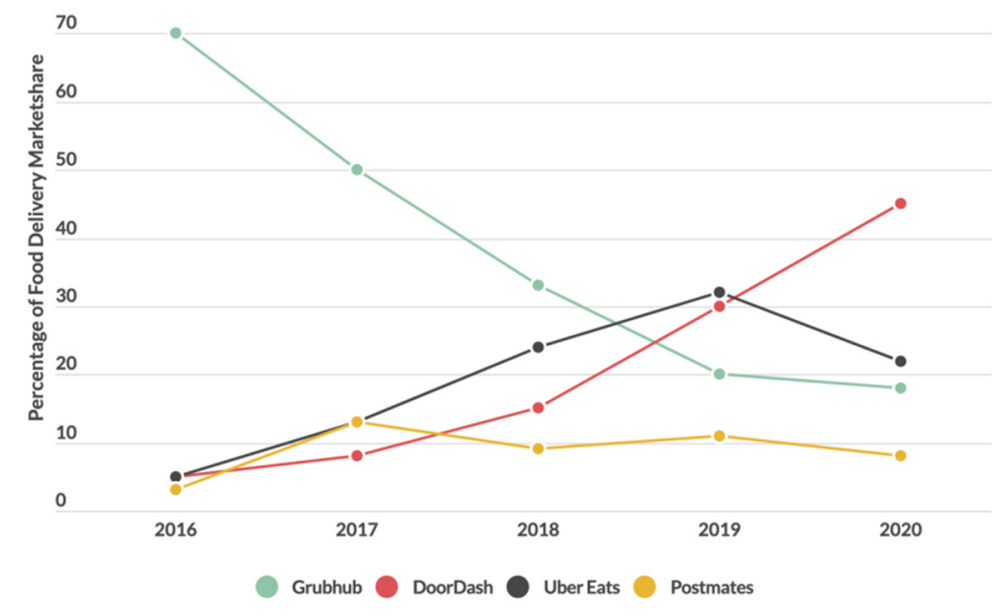

Figure 2: An image that explicitly shows how these major existing companies compete fiercely from 2016 to 2020. Source. Business of Apps.

Figure 2 reflects the rapid increasing speed of DoorDash in five years. Nevertheless, the rise of DoorDash leads to the fact that other companies lost their market shares and the fact that the competition in the food delivery industry became more fierce.

\section{THREAT OF NEW ENTRANTS}

According to the intense competition in the food delivery industry, the threat of new entrants is quite low. From the aspect of customer loyalty, at present, there are four major companies that occupy almost all the delivery orders, which are DoorDash, Grubhub, Uber
Eats, and Postmates which was bought by Uber Eats in 2020, and DoorDash offers half of these orders. If a new company attempts to entry in this industry, it will face barriers to entry. High start-up costs are the first problem that a newcomer must consider. After investing a myriad of money, a newcomer needs to figure out the customer problem. For the reason that customers have been familiar with those long existing food delivery platforms, a newcomer will have barriers to entry in brand identity and customer loyalty. Another aspect is the concern of restaurant platforms. Restaurants may earn less profits in accompanying with food delivery companies, as expanding direct food delivery services 
by themselves reduces the commission fees. Moreover, food delivery companies have been utilized the explosion of orders during pandemic to make profits and to accelerate their growth. It's hard for new entrants to hold the pandemic benefits. Consequently, the possibility for the companies which attempt to enter the food delivery industry is small.

\section{THREAT OF SUBSTITUTE PRODUCTS}

As demonstrated by the existing companies in this industry, the threat of substitute products is large. In the food delivery industry, there are several companies doing the relatively same platforms and service, and even many restaurants on different platforms are the same. The following statistics in Figure 3 show the percentages that customers used different food delivery platforms simultaneously in the first quarter of 2021. The data in the DoorDash column shows that there is about $1-43 \%$ (which equals $57 \%$ ) of people are loyal to DoorDash in the first quarter of 2021. Compared to Grubhub, Uber Eats, and Postmates, DoorDash has the highest customer loyalty, which is a positive sign that although there are several substitute products in the market, about half of the customers would like to choose DoorDash instead of others.

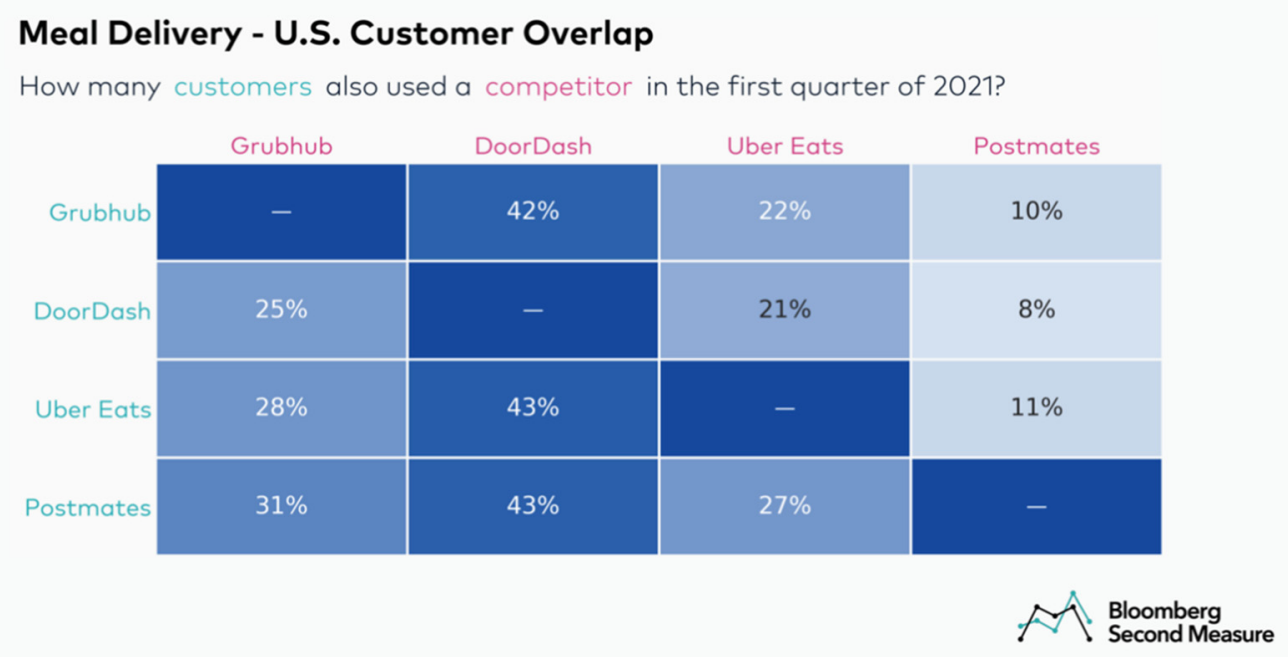

Figure 3: A table that contains the data in the field of customer loyalty in the first quarter of 2021.

Source. Bloomberg Second Measure.

Although in 2021, DoorDash "went public on the New York Stock Exchange at a $\$ 72$ billion valuation" [1], this company hasn't made any profit until the second quarter of 2020, and yet, it has been still posting no profit. DoorDash has been "raising and losing billions of dollars to subsidize prices to gain market share" [2] for years, and substitute products can limit the profit potential of an industry. To gain more market share, companies can only drive down the price, which could result in the rising of the orders, but it could also result in low revenues, which is a difficulty for DoorDash or any other developing companies. Recently, DoorDash has been "diversifying its offerings in recent months by partnering with grocery stores and convenience stores" [3]. But there are competitors as well, for instance, the grocery delivery company Instacart. DoorDash also has partnerships with CVS for the delivery of household essentials.

\section{BARGAINING POWER OF BUYERS}

For DoorDash, the bargaining power of buyers is relatively weak in American suburbia. Although metropolises have more potential customers with disposable income and more restaurants that customers could choose, DoorDash chose to develop in small areas, and other online ordering platforms haven't been involved. DoorDash noticed that on-demand delivery hasn't been served in small areas. Therefore, residents in the country are affected by lacking substitutes and long driving distances to merchants. For this reason, those people could receive large benefits from ondemand delivery, and they could be more dependent on online ordering. For DoorDash, developing in the suburbia has benefits in light traffic and easy parking, which could enable the delivery drivers to become more efficient. Moreover, the price of orders from the customers in small areas could be higher for the reason that each order could be ordered for a whole family, instead of an individual. Also, since there are fewer online ordering applications, those people could be more loyal to one platform. "DoorDash says they have a $58 \%$ market share in the suburbs, higher than their overall market share" [4]. As a consequence, in American suburbia, the bargaining power of buyers is weak. 


\section{BARGAINING POWER OF SUPPLIERS}

In the fierce competition in the food delivery and online ordering industry, the bargaining power of suppliers became stronger than before. Since quarantine was required during the pandemic, all the restaurants were forced to close for months, even a year. This situation resulted in a phenomenon that many restaurants closed down and the rising of food delivery services. Hence, many restaurants chose to accompany food delivery platforms to make a living. However, taking $15 \%$ to $30 \%$ of commission fees for every delivery order is taking away a great part of profits from the original tiny profits. DoorDash, like other food delivery platforms, has "come under increased scrutiny during the pandemic for the fees it charges restaurants" [5]. To solve the commission fee issue, California has limited fees at $15 \%$ of the menu-listed price of the order. After that, DoorDash now offers different levels of commission fees on deliveries, and higher commission fees offer more beneficial service. Some restaurants provide direct orders to avoid the high commission fees. Also, after quarantine, when restaurants don't have to depend on delivery service, the bargaining power of suppliers will become much stronger.

\section{CONCLUSION}

Consequently, for DoorDash, it has superiority in rivalry among existing competitors in the industry because its rapid increasing speed in three years. The threat of substitute products of DoorDash is strong, for the reason that it has the highest customer loyalty in the food delivery industry. Also, the bargaining power of suppliers is becoming stronger. It lowered the commission fees. Meanwhile, the threat of new entrants is small because the food delivery industry has been crowded and competitive. New entrants lack of market and demands. The bargaining power of buyers is relatively weak in the American suburbia, but in some metropolises, DoorDash does not have stronger bargaining power of buyers than other online ordering and food delivery companies.

\section{ACKNOWLEDGMENTS}

I would like to express my deep gratitude to Professor Honigsberg and my research supervisors, for their patient guidance, enthusiastic encouragement and useful critiques of this research work. My grateful thanks are also extended to my teacher assistant Yoki, who generously gave me her advice and assistance in keeping my progress on schedule. Finally, I wish to thank my parents for their support and encouragement throughout my study.

\section{REFERENCES}

[1] Curry, David. Business of Apps. (2021) DoorDash Revenue and Usage Statistics (2021). https://www.businessofapps.com/data/doordashstatistics/

[2] Brien, Sara Ashley O.. CNN Business. (2020) DoorDash Soars $85 \%$ in Wall Street Debut. https://edition.cnn.com/2020/12/09/tech/doordashipo/index.html

[3] CNN Business. (2020) Food Delivery Giant DoorDash Files Paperwork to Go Public. https://edition.cnn.com/2020/11/13/tech/doordashipo-paperwork/index.html

[4] Kamp, Lars. Medium. (2020) How DoorDash Built the Most Incredible Go-to-market Playbook Ever. https://larskamp.medium.com/how-doordash-builtthe-most-incredible-go-to-market-playbook-ever$5 \mathrm{e} 8 \mathrm{f} 1 \mathrm{~d} 58 \mathrm{f} 6 \mathrm{~cd}$

[5] Lucas, Amelia. CNBC. (2020) DoorDash Scores Valuation of \$16 Billion As Coronavirus Pushes It to Top of Food-Delivery Chain. https://www.cnbc.com/2020/06/19/doordashscores-16-billion-valuation-now-top-of-fooddelivery-chain.html 